\title{
Scanning Photo-Induced Impedance Microscopy-Resolution studies and polymer characterization
}

\author{
Steffi Krause ${ }^{\mathrm{a}, *}$, Werner Moritz ${ }^{\mathrm{b}}$, Habib Talabani $^{\mathrm{c}}$, Ming Xu ${ }^{\mathrm{c}}$, Andrea Sabot ${ }^{\mathrm{c}}$, Graham Ensell ${ }^{\mathrm{d}}$ \\ ${ }^{a}$ Department of Materials, Queen Mary, University of London, London E1 4NS, UK \\ ${ }^{\mathrm{b}}$ Humboldt University Berlin, Brook-Taylor-Str. 2, 12489 Berlin, Germany \\ ${ }^{\mathrm{c}}$ Department of Chemistry, University of Sheffield, Sheffield S3 7HF, UK \\ ${ }^{\mathrm{d}}$ Department of Electronics and Computer Science, University of Southampton, SO17 1BJ, UK
}

Received 23 June 2004; received in revised form 25 October 2004; accepted 25 February 2005

Available online 22 August 2005

\begin{abstract}
Scanning Photo-Induced Impedance Microscopy (SPIM) is an impedance imaging technique that is based on photocurrent measurements at field-effect structures. The material under investigation is deposited onto a semiconductor-insulator substrate. A thin metal film or an electrolyte solution with an immersed electrode serves as the gate contact. A modulated light beam focused into the space charge region of the semiconductor produces a photocurrent, which is directly related to the local impedance of the material. The absolute impedance of a polymer film can be measured by calibrating photocurrents using a known impedance in series with the sample.

Depending on the wavelength of light used, charge carriers are not only generated in the focus but also throughout the bulk of the semiconductor. This can have adverse effects on the lateral resolution. Two-photon experiments were carried out to confine charge carrier generation to the space charge layer. The lateral resolution of SPIM is also limited by the lateral diffusion of charge carriers in the semiconductor. This problem can be solved by using thin silicon layers as semiconductor substrates. A resolution of better than $1 \mu \mathrm{m}$ was achieved using silicon on sapphire (SOS) substrates with a $1 \mu \mathrm{m}$ thick silicon layer.
\end{abstract}

(C) 2005 Elsevier Ltd. All rights reserved.

Keywords: Localized impedance; Imaging; Resolution; Two-photon; LAPS; Polymers

\section{Introduction}

Electrochemical impedance spectroscopy as a macroscopic technique has the disadvantage that the data obtained always represents the properties of the structure under investigation averaged across the whole sample area. To investigate the behaviour of heterogeneous systems, impedance measurements with good lateral resolution are desirable. In recent years, different techniques capable of providing information about the local impedance have been proposed. One of the most successful approaches to date is local electrochemical impedance spectroscopy (LEIS). It uses a two-electrode probe to measure the local current density close to the sur-

\footnotetext{
* Corresponding author. Tel.: +44 207 8823747; fax: +44 2089819804.

E-mail address: s.krause@qmul.ac.uk (S. Krause).
}

face of the working electrode $[1,2]$. Problems associated with the technique are limited miniaturization of the two-electrode probe, that only current densities normal to the surface are considered and that there is no feedback mechanism inherent in the technique that allows positioning of the probe at a defined distance from the surface. Lohrengel et al. developed a technique that provides a very well-defined measurement area $[3,4]$. Glass capillaries with a silicone rubber gasket at their end (diameter $>10 \mu \mathrm{m}$ ) are used to position electrolyte droplets onto the surface of the working electrode. The smallest usable measurement area was estimated to have a diameter of about $6 \mu \mathrm{m}$ depending on the impedance of the substrate [4]. This technique is particularly useful for the characterization of solid surfaces where information about inclusions, grain boundaries and grain-dependent passivation is sought. Katemann et al. proposed to use an AC scanning electrochem- 
ical microscopy (SECM) setup to image the impedance of a substrate surface [5]. At low electrolyte concentrations and sufficiently high frequency the contribution of the doublelayer capacitance of the tip to the total impedance of the system is negligible and the tip current magnitude is strongly associated with the solution resistance and the conductivity of the electrode surface. Compared to LEIS, this technique has potential for improving the spatial resolution by using smaller microelectrodes as scanning probes.

\subsection{Scanning Photo-Induced Impedance Microscopy (SPIM)}

The localized impedance techniques described above are all based on scanning microelectrode probes. A different approach based on scanning a laser beam across a semiconductor substrate was developed by Krause et al. [6]. The technique, Scanning Photo-Induced Impedance Microscopy, is specifically aimed at the investigation of the local dielectric properties of thin films, membranes and biological materials. A film of the material under investigation is deposited onto a semiconductor/insulator substrate. A gate contact on the material is created by depositing a thin metal layer onto the film or by exposing the material to an electrolyte solution. In the latter case, the circuit would be completed with an electrode immersed in the solution. A bias is applied between the gate electrode and the semiconductor substrate to create an inversion layer at the semiconductor/insulator interface. Light focussed into the space charge layer of the semiconductor generates electron-hole pairs that separate in the field of the inversion layer causing a current to flow in the outer circuit. Electron-hole pairs generated by the light in the bulk of the semiconductor can also contribute to the current if they diffuse into the space charge region. The current is confined to the illuminated area of the semiconductor and provides information about the local impedance of the material deposited onto the structure. Modulation of the light at different frequencies allows measurement of impedance spectra as will be demonstrated using cellulose acetate film as an example (see Section 3.3).

\subsubsection{Resolution of SPIM}

In terms of experimental approach and substrate materials, SPIM is very closely related to Light Addressable Potentiometric Sensors (LAPS) and the Scanned Light Pulse Technique (SLPT) [7-9]. While SPIM detects photocurrent changes with a metal-insulator-semiconductor (MIS) or an electrolyte-insulator-semiconductor (EIS) structure biased towards inversion (saturation region of the photocurrent-voltage curve) to detect local changes in the impedance, LAPS and SLPT determine shifts of the current-voltage characteristic along the DC voltage axis at a bias near the flat band point to detect local changes in potential. Hence, results obtained regarding the resolution can easily be transferred from one technique to the other.
Factors that can influence the resolution of all three techniques are the quality of the focus in the space charge layer of the semiconductor, the scattering of light within the structure and the diffusion of charge carriers. Electron-hole pairs generated in the bulk of the semiconductor do not only diffuse towards the space charge region in the semiconductor where they produce a current, but they also diffuse laterally, out of the illuminated area, resulting in poor resolution. Two main strategies have been pursued to improve the lateral resolution of SPIM and LAPS. The traditionally used single crystalline silicon can be replaced with semiconductors that have shorter charge carrier life times. Promising results have been obtained using GaAs [10]. The effective diffusion length was determined to be about $3 \mu \mathrm{m}$ in a $500 \mu \mathrm{m}$ thick substrate. Unfortunately, the problem of a high quality gate insulator on GaAs with low leakage current has not been solved yet. Another promising material is amorphous silicon [11]. Films of the material with a thickness ranging from 0.3 to $1.3 \mu \mathrm{m}$ were deposited onto a glass substrate with a thin film of $\mathrm{ZnO}$ as the ohmic contact. $\mathrm{SiO}_{2} / \mathrm{Si}_{3} \mathrm{~N}_{4}$ films were deposited as the gate insulator. The effective diffusion length in this arrangement was too short to be measured with the experimental setup used (i.e. $<100 \mathrm{~nm}$ ). Hence submicrometer resolution of photocurrent measurements is possible using this material if a good quality optical setup is used. However, problems caused by multiple reflections in the multilayer structure used for these experiments have yet to be solved.

Another strategy for improving the resolution involves the use of a thin silicon substrate. Theoretical considerations and experimental results described in the literature suggest that the resolution of photocurrent measurements is closely related to the thickness of the silicon $[12,13]$. Nakao et al. achieved a resolution of about $10 \mu \mathrm{m}$ for LAPS using a $20 \mu \mathrm{m}$ thick silicon substrate [12]. Ito reported a resolution of $5 \mu \mathrm{m}$ obtained with a $0.5 \mu \mathrm{m}$ thick silicon layer [14]. However, in this case, the resolution was determined by the size of differently doped islands in the silicon resembling a microelectrode array. Hence, a conclusion about the maximum possible resolution could not be drawn from these experiments.

In this paper, two strategies for improving the resolution were investigated more closely.

1. To carry out SPIM measurements, it is necessary to focus light into the space charge region of the semiconductor from the back of the substrate, i.e. while light is travelling through the semiconductor, charge carriers are produced throughout the bulk of the silicon and not only in the space charge region (Fig. 1A). Two photon experiments have been used in the past to characterise electrical circuits using a technique called two-photon optical beam induced current imaging (TOBIC) $[15,16]$. In this case, light of energy smaller than the bandgap of silicon is used. Using high intensity illumination by focusing a beam from a high energy pulsed laser delivering femtosecond bursts at high frequencies, two photons can arrive almost simultaneously and combine to generate electron hole 

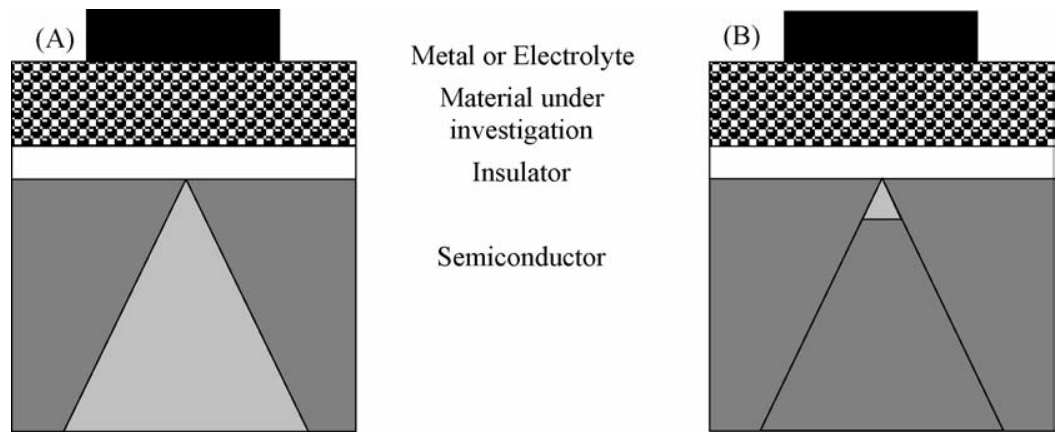

Fig. 1. (A) In case of a single photon effect charge carriers are generated throughout the bulk of the semiconductor. (B) In case of the two-photon effect charge carrier generation is confined to a small volume close to the focus.

pairs. Since the absorption rate and therefore the photocurrent increases quadratically with the local light intensity, charge carrier generation is confined to the vicinity of the focal point, i.e. no charge carrier generation will be observed in the bulk of the semiconductor (Fig. 1B).

2. SPIM measurements were carried out with thin silicon layers as semiconductor substrates. The results obtained with silicon on insulator (SOI) and silicon on sapphire (SOS) substrates will be compared.

\section{Experimental}

\subsection{Sample fabrication}

The following semiconductor substrates were used:

- double polished silicon (thickness $400 \mu \mathrm{m}$, boron doped, $10-30 \Omega \mathrm{cm})$;

- silicon on insulator (Analog Devices, Belfast) with a $7 \mu \mathrm{m}$ thick device layer (boron doped, $1-3 \Omega \mathrm{cm}$ ), a $1 \mu \mathrm{m}$ thick buried oxide and a $400 \mu \mathrm{m}$ thick handle;

- silicon on sapphire (PB Technik) with a $1 \mu \mathrm{m}$ thick silicon layer (boron doped, $0.1 \Omega \mathrm{cm}$ ) on a $475 \mu \mathrm{m}$ thick sapphire substrate.

A gate oxide was thermally grown on all semiconductor substrates $(40 \mathrm{~nm}$ on double polished silicon and SOI, $70 \mathrm{~nm}$ on SOS). A $2.7 \mathrm{~mm} \times 2.7 \mathrm{~mm}$ window was etched by DRIE into the handle of the SOI wafer. The buried oxide remaining after DRIE was removed using $10 \% \mathrm{HF}$ to allow focusing of the laser onto the device layer.

In order to form an ohmic contact to each of the silicon substrates, part of the thermally grown oxide was removed from the device side of the samples using $10 \% \mathrm{HF}$. Some $100 \mathrm{~nm}$ of Al was thermally evaporated onto the etched areas. The samples were then heated to $350^{\circ} \mathrm{C}$ for $5 \mathrm{~min}$ to form the ohmic contact.

To measure the effective diffusion length in the semiconductor materials used, a gate electrode with a clearly defined edge had to be fabricated. For that purpose, $10 \mathrm{~nm}$ of chromium and $40 \mathrm{~nm}$ of gold were thermally evaporated onto the gate oxide and patterned by lift-off using a standard photolithographic technique.

SOI substrates were also used for the characterisation of polymers in solution. Polymethylmethacrylate (MW $=75,000$, Aldrich, UK) was coated from a $4 \%$ solution of the polymer in $m$-xylene by depositing a droplet of the polymer on the gate oxide and letting it dry. Cellulose acetate films were spin-coated from a $5 \%$ solution of the polymer in acetone at $1000 \mathrm{rpm}$ for $60 \mathrm{~s}$.

\subsection{Photocurrent measurements}

The experimental setup for photocurrent measurements (shown in Fig. 2) consisted of an EG\&G 7260 lock-in amplifier, a laser, an electro-optic modulator, a beam expander, a $50 \times$ microscope objective (Olympus, SLMPlanFl50) and an $X Y Z$ positioning system (Physik Instrumente). The control software was written in house using LabView. All samples were biased towards inversion during the measurements. For the two-photon experiments described in Section 3.1, a Femtolite Supercontinuum laser from IMRA Lasers (port 2 option, $1560 \mathrm{~nm}, 60 \mathrm{~mW}$, <400 fs, $80 \mathrm{MHz}$ ) was used. SPIM experiments in electrolyte contact (Section 3.3) were carried out using a He-Ne Laser (632 nm, $5 \mathrm{~mW}$ ).

For measurements of the diffusion length of charge carriers (Section 3.3), the setup was modified as follows. A titanium-sapphire laser operated at $76 \mathrm{MHz}$ was utilized as the light source. In conjunction with a frequency doubler, the setup produced a final wavelength of $430 \mathrm{~nm}$ at an average power of $1 \mathrm{~mW}$. The beam was focused onto a pinhole and then collimated to obtain a perfectly Gaussian laser beam profile. The beam was then chopped and focused onto the sample using a microscope objective $(100 \times / 0.9)$.

For measurements of the diffusion length, test structures were mounted onto the positioning system with the gate electrode facing the laser beam. The laser was focused and scanned across the metal edge as shown in Fig. 3. While the laser is focused onto the metal, only a small photocurrent flows since most of the light is absorbed by the metal. When the laser beam leaves the metal, a sudden increase in the photocurrent is observed. The current decays, as the light 


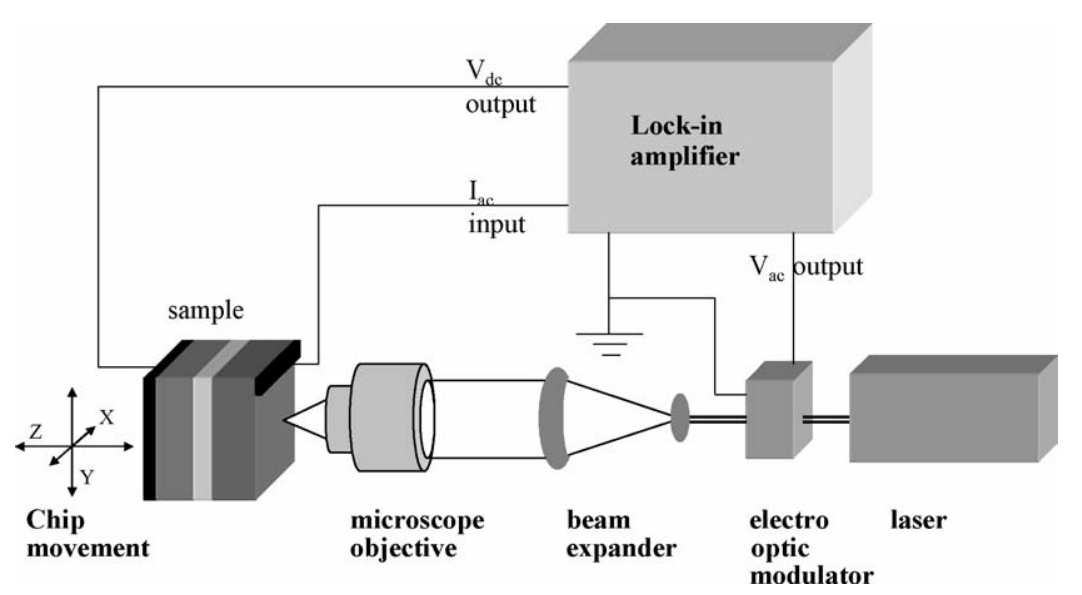

Fig. 2. Experimental setup for photocurrent measurements.

moves further away from the edge. The length of the decay is a measure for the lateral diffusion of charge carriers.

For the characterisation of polymers in electrolyte contact, the samples were mounted onto the positioning system with the back of the semiconductor facing the laser beam. Measurements were carried out in a $0.1 \mathrm{M} \mathrm{Na}_{2} \mathrm{SO}_{4}$ solution in purified water. A printed carbon electrode was used as the gate electrode to minimise reflections of the laser from the gate electrode back into the sample. The wetted sample area was about $7 \mathrm{~mm}^{2}$. The sample holder for electrolyte measurements has been described elsewhere [6].

\subsection{Impedance measurements}

High-frequency capacitance-voltage (HF-CV) and ACimpedance measurements were carried out using an Autolab Frequency Response Analyser FRA2 with a potentiostat PGSTAT10 (Windsor Scientific, UK). An AC voltage with an amplitude of $10 \mathrm{mV}$ superimposed on a DC voltage was applied between gate contact and ohmic backside contact of the field effect structures. From HF-CV measurements a DCbias for AC-impedance measurements in accumulation was chosen. AC impedance measurements were carried out at a constant DC-voltage over a frequency range from $10 \mathrm{~Hz}$ to $10 \mathrm{kHz}$

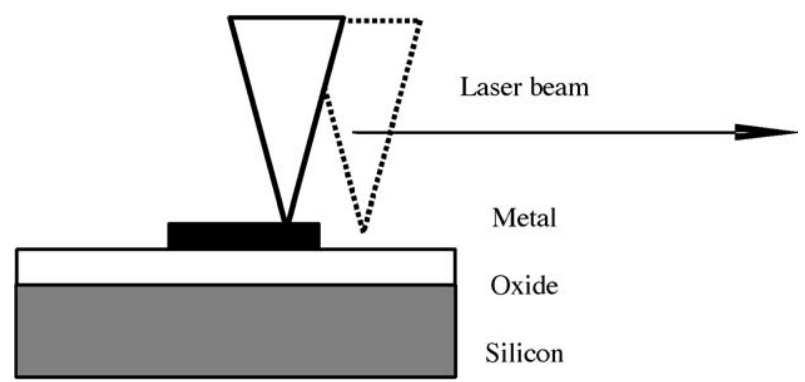

Fig. 3. The effective diffusion length was measured by scanning a focused laser beam across the edge of the gate metal.

\section{Results}

\subsection{Effective diffusion length of charge carriers using a two-photon effect in silicon}

By using light with an energy smaller than the bandgap in silicon and relying on a two-photon effect to generate charge carriers, charge carrier generation can be confined to the space charge layer in silicon (see Section 1). Hence, an improved resolution of photocurrent measurements at bulk silicon was expected. To demonstrate that a two-photon effect was indeed obtained, the intensity dependence of the photocurrent was measured using the femtosecond laser at $1560 \mathrm{~nm}$ and a He-Ne Laser at $632 \mathrm{~nm}$, the latter of which produced an ordinary single photon effect. The double logarithmic plot of photocurrent versus intensity shows a linear relationship in both cases (Fig. 4). A slope close to 1 was obtained for the $\mathrm{He}-\mathrm{Ne}$ laser indicating a linear relationship between current and intensity as expected for a single photon experiment. In the case of the longer wavelength, a slope of 2.04 was obtained confirming that the photocurrent increases quadratically with the intensity as expected for a two-photon experiment.

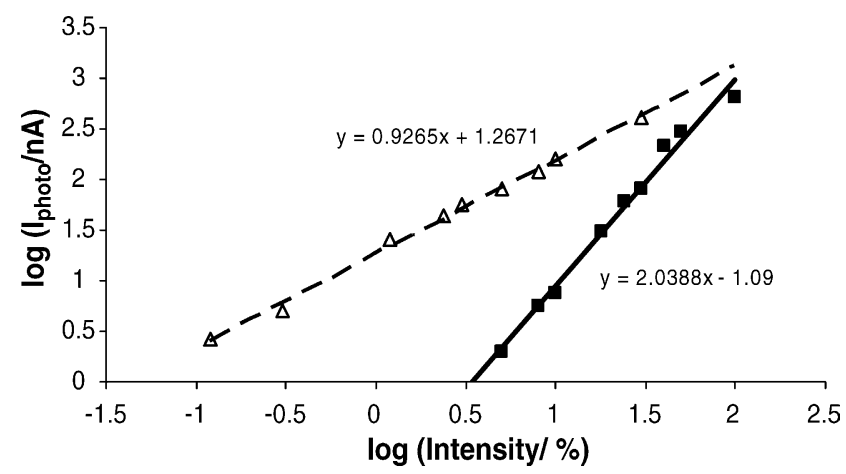

Fig. 4. Intensity dependence of the photocurrent using a femtosecond laser with a wavelength of $1560 \mathrm{~nm}$ (squares) and a He-Ne laser (triangles). 


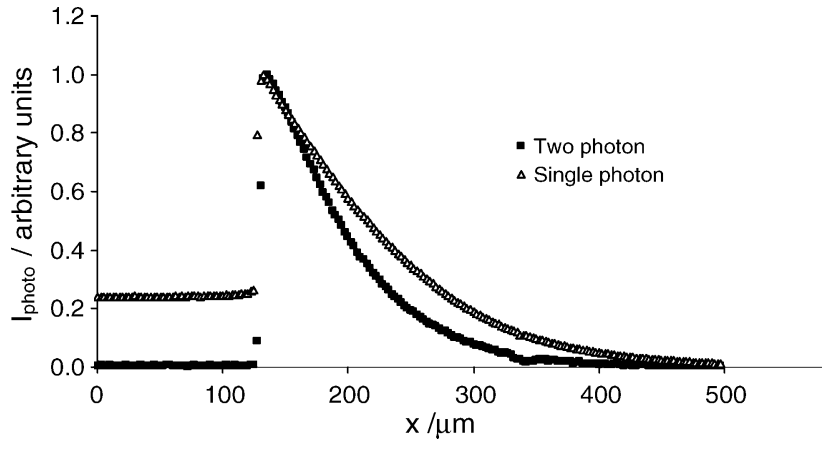

Fig. 5. Photocurrent line scan across the edge of the gate metal on a $\mathrm{Si} / \mathrm{SiO}_{2} / \mathrm{Cr} / \mathrm{Au}$ structure using a femtolite laser $(1560 \mathrm{~nm})$ and a $\mathrm{He}-\mathrm{Ne}$ Laser $(632 \mathrm{~nm})$.

To compare the resolution that can be achieved with single and two-photon experiments, the effective diffusion length of charge carriers was measured by scanning both lasers across a metal edge as described above (see Fig. 3). For the single photon experiment, a diffusion length of $112 \mu \mathrm{m}$ was determined, and for the two-photon experiments, the diffusion length was $78 \mu \mathrm{m}$ (Fig. 5). This corresponds to an improvement in the resolution by $31 \%$. Unfortunately, the improvement is still not sufficient to allow high resolution photocurrent measurements using bulk silicon.

\subsection{Effective diffusion length of charge carriers in thin silicon layers}

Nakao et al. achieved a resolution of $10 \mu \mathrm{m}$ using a $20 \mu \mathrm{m}$ thick silicon substrate [12]. Measurements of the effective diffusion length were carried out using a $7 \mu \mathrm{m}$ thick silicon layer on an SOI substrate and a $1 \mu \mathrm{m}$ thick layer on an SOS substrate to find out whether this resolution could be improved by using even thinner silicon layers. Fig. 6 shows a line scan over the gate edge at a field-effect structure using an SOI substrate (see inset in Fig. 6). A diffusion length of $13 \mu \mathrm{m}$ was determined.

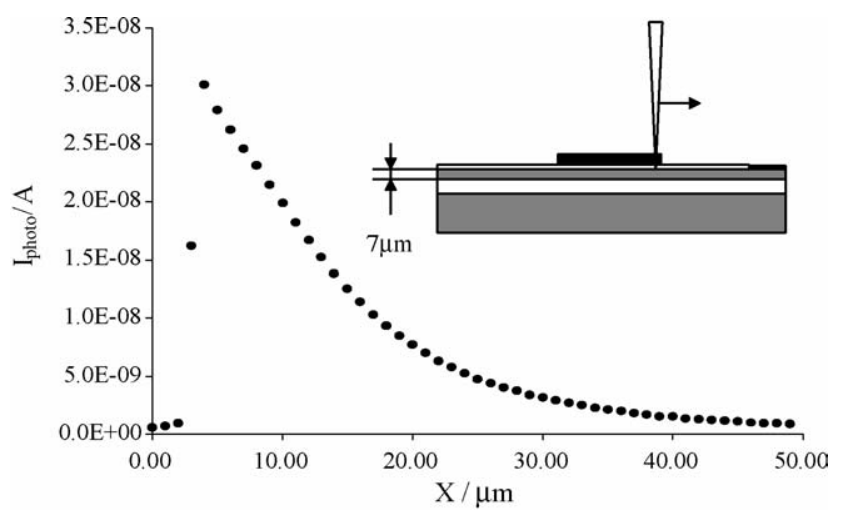

Fig. 6. Photocurrent line scan across the edge of the gate metal on an $\mathrm{SOI} / \mathrm{SiO}_{2} / \mathrm{Cr} / \mathrm{Au}$ structure using a Ti-sapphire laser with frequency doubler $(430 \mathrm{~nm})$. The sample structure is shown in the inset.

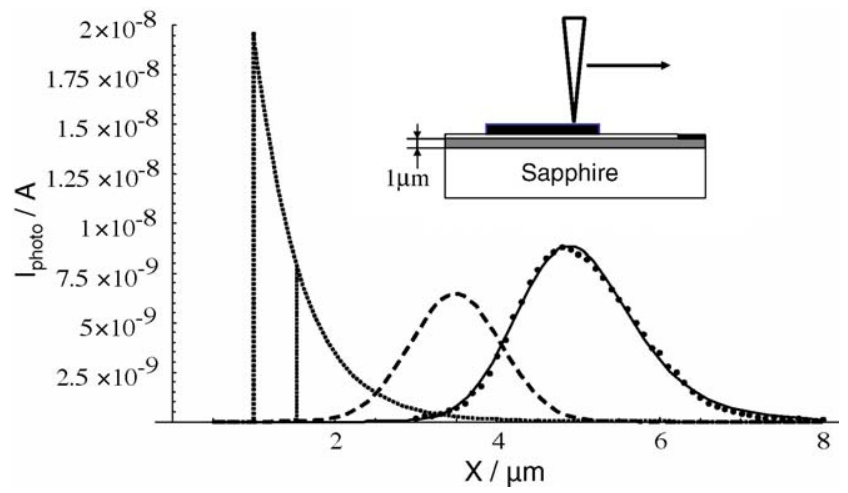

Fig. 7. Photocurrent line scan across the edge of the gate metal on an $\mathrm{SOS} / \mathrm{SiO}_{2} / \mathrm{Cr} / \mathrm{Au}$ structure using a Ti-sapphire laser with frequency doubler $(430 \mathrm{~nm})$. Measured data appear as dots and the fitted curve as solid line. The results of the deconvolution, the exponential decay and the Gauss profile, are represented as dotted and broken lines, respectively. The sample structure is shown in the inset.

The results obtained using SOS substrates are shown in Fig. 7. In this case, the photocurrent decays over a distance of about $3 \mu \mathrm{m}$. In all other measurements, the initial increase of the photocurrent as the laser spot moves across the edge of the metal was very steep compared to the exponential decay (Figs. 5 and 6). However, in case of the SOS substrate, the rise of the photocurrent occurs over a similar distance as its decay, i.e. the profile of the laser beam and the exponential decay due to the diffusion of charge carriers are convoluted. To determine the effective diffusion length of the SOS based samples, a deconvolution of the line scan had to be carried out. This was achieved by fitting the line scan to a convolution integral assuming a Gauss distribution for the laser beam profile and a simple exponential decay for the diffusion of charge carriers. The results of the deconvolution are plotted in Fig. 7. From the Gauss profile a laser spot diameter of $1.3 \mu \mathrm{m}$ and from the exponential decay an effective diffusion length of $0.57 \mu \mathrm{m}$ were calculated. This confirms that SPIM and LAPS measurements with submicrometer resolution are possible using thin, single crystalline films of silicon on sapphire.

\subsection{SPIM measurements at polymer films}

\subsubsection{Spatially resolved measurements}

To compare the resolution estimated from diffusion length measurements with the resolution achieved using SPIM, a polymer dot was deposited onto the gate area of a SOI/SiO structure and the sample exposed to an electrolyte solution (Fig. 8A). As these measurements were to simulate realistic conditions for SPIM measurements, the laser was focused from the back of the semiconductor substrate. Fig. 8B shows an area scan of the structure. The polymer dot is clearly visible as a reduction in photocurrent in the coated area. From a photocurrent line scan across the edge of the film (Fig. 8C) a resolution of about $24 \mu \mathrm{m}$ can be estimated. This value is in 
(A)

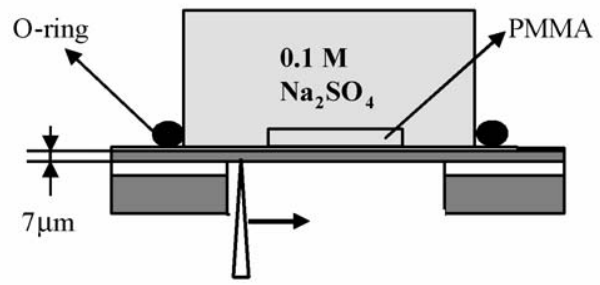

(B)
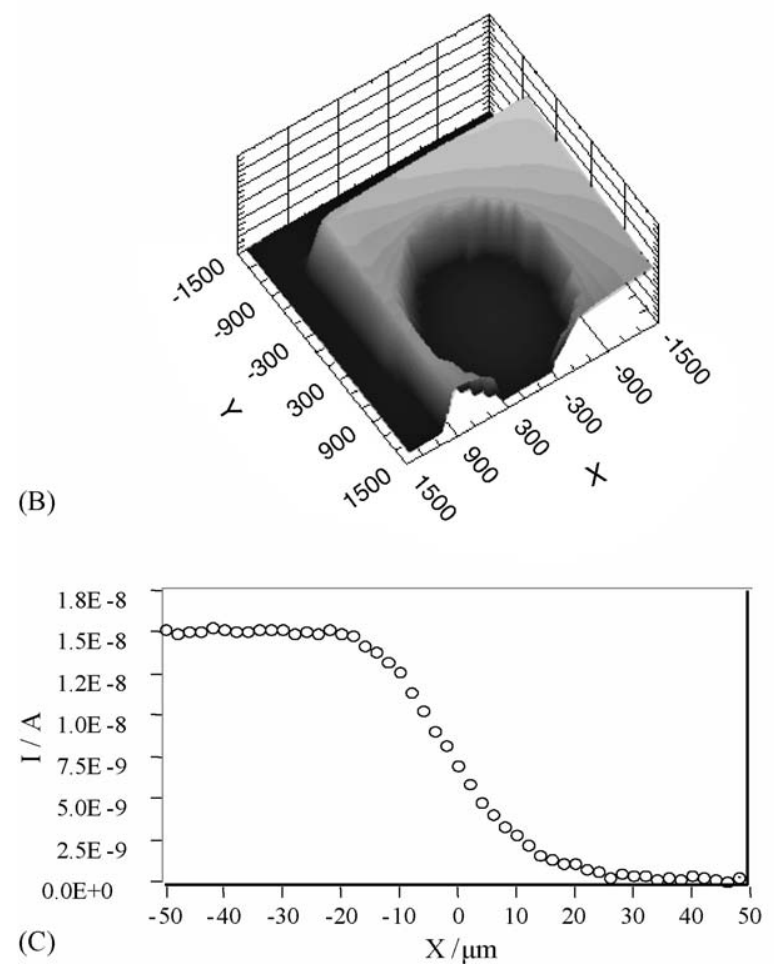

Fig. 8. (A) PMMA dotted onto the gate area of a $\mathrm{SOI} / \mathrm{SiO}_{2}$ structure with a $7 \mu \mathrm{m}$ thick silicon membrane. The gate area was exposed to an $0.1 \mathrm{M}$ $\mathrm{Na}_{2} \mathrm{SO}_{4}$ solution; (B) photocurrent area scan of the PMMA dot (scale in $\mu \mathrm{m})$; (C) photocurrent line scan across the edge of the PMMA dot.

the same order of magnitude as, but also somewhat larger than the diffusion length of $13 \mu \mathrm{m}$ (see Section 3.2). A possible reason for this discrepancy is the optical setup used in these experiments. Diffusion length measurements were carried out with blue light at a wavelength of $430 \mathrm{~nm}$. The penetration depth of light of this wavelength is very small. Since the laser was focused onto the front of the structure, most of the light would be absorbed in the space charge layer of the semiconductor. In the measurements shown in Fig. 8, a red laser $(632 \mathrm{~nm})$ was focused onto the back of the sample, i.e. charge carriers were produced throughout the bulk of the semiconductor due to the direction the laser light came from and due to the greater penetration depth of the red laser. The latter can also lead to reflections of light from the front of the sample back into the semiconductor causing a further broadening of the signal. Another reason for the discrepancy may be the quality of the polymer edge, as this was not created with a lithographic method.

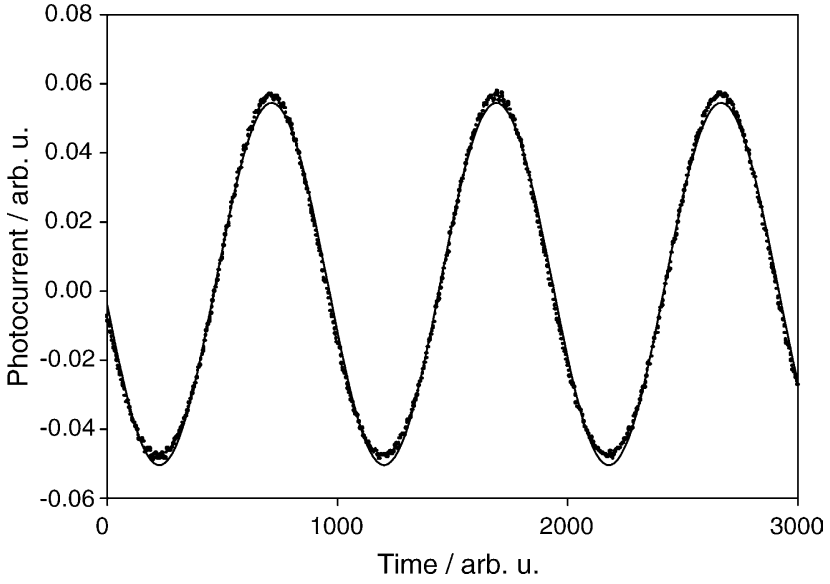

Fig. 9. Time dependence of the photocurrent at a frequency of $1 \mathrm{kHz}$. The solid line represents the fit of the trace to a sine function.

\subsubsection{Impedance measurements using SPIM}

Linearity of photocurrent and excitation signal is important if meaningful impedance data are to be obtained using SPIM. The laser intensity is modulated using an electro-optic modulator with a sinusoidal input signal. For all experiments a modulation depth $<20 \%$ was chosen. The time dependent signal of the photocurrent measured at $1 \mathrm{kHz}$ shows that the condition of linearity is fulfilled since the photocurrent trace can be fitted to a sine function (Fig. 9).

In ref. [6], impedance data obtained from standard ACimpedance measurements and SPIM were compared for an insulating polymer. In the following, it will be demonstrated that more complex impedance behaviour can also be measured using SPIM. Cellulose acetate was chosen as the model system as it is known to take up well defined amounts of electrolyte [17]. To compare SPIM results with AC-impedance data, the entire sample was coated with cellulose acetate. The same measurement area for AC-impedance measurements and SPIM was guaranteed by moving the sample out of focus for photocurrent measurements to illuminate the entire wetted area.

A simple way to calibrate photocurrent measurements is to measure the photocurrent with and without a known impedance in series with the sample. For the experiments presented here an $R C$ parallel combination $(0.8 \mathrm{M} \Omega, 1 \mathrm{nF})$ was chosen as the calibration circuit. The expressions for the impedance with and without calibration circuit are given in Eqs. (1) and (2):

$\frac{V_{\text {photo }}}{I_{\text {photo }}^{\text {sample }}}=Z_{\text {sample }}$

$\frac{V_{\text {photo }}}{I_{\text {photo }}^{\text {sample }+R C}}=Z_{\text {sample }}+Z_{R C}$

where $V_{\text {photo }}$ is the photovoltage, $I_{\text {photo }}^{\text {sample }}$ the photocurrent without calibration circuit, $I_{\text {photo }}^{\text {sample }+R C}$ the photocurrent with calibration circuit, $Z_{\text {sample }}$ the impedance of 

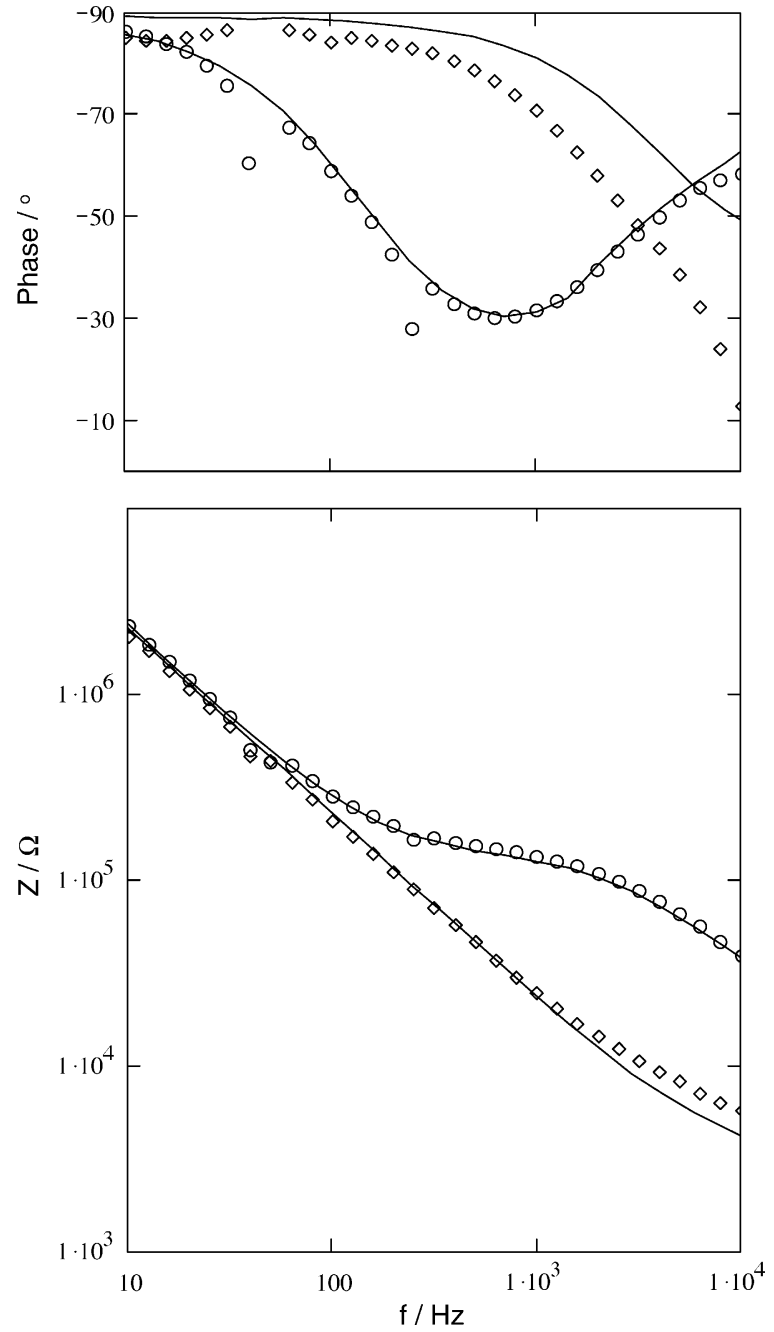

Fig. 10. Comparison of impedance spectra derived from SPIM (represented by dots) and impedance spectra measured by classical $\mathrm{AC}$-impedance spectroscopy (represented by lines). The impedance spectra of an uncoated structure (diamonds) and a structure coated with cellulose acetate (circles) are shown.

the sample, and $Z_{R C}$ is the impedance of the calibration circuit.

Assuming that the photovoltage is the same in both cases, the impedance of the sample can be calculated from

$Z_{\text {sample }}=\frac{Z_{R C} \times I_{\text {photo }}^{\text {sample }+R C}}{I_{\text {photo }}^{\text {sample }}-I_{\text {photo }}^{\text {sample }+R C}}$

The impedance spectra obtained from SPIM and ACimpedance measurements for an uncoated structure and a structure coated with cellulose acetate are presented in Fig. 10. The spectra measured with both techniques show good agreement. Slightly larger deviations were observed for the spectra of the uncoated structure. This maybe due to a small leakage current caused by pin holes in the silicon dioxide layer. DC-currents flowing through the structure during photocurrent measurements are known to cause experimental error. The effect would be less pronounced for a polymer

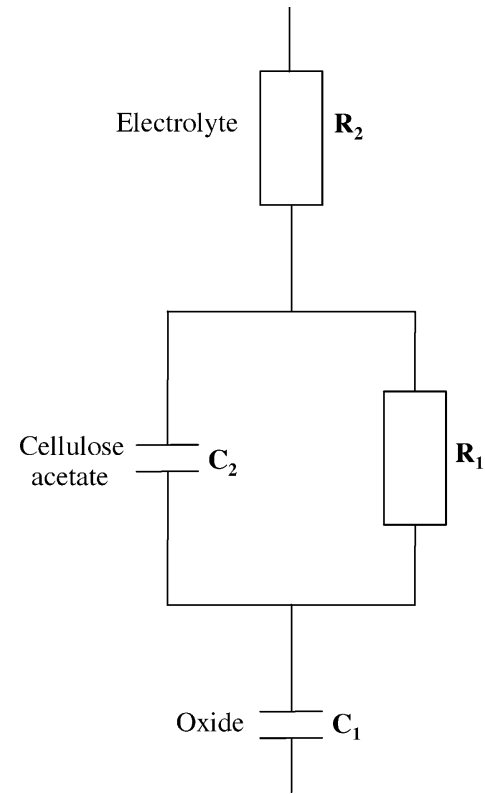

Fig. 11. Equivalent circuit for a $\mathrm{SOI} / \mathrm{SiO}_{2} /$ cellulose acetate/electrolyte structure.

coated structure as any existing pin-holes would be covered up by the polymer.

The impedance spectra of the uncoated structure were fitted with a resistor and a capacitor in series, representing the electrolyte resistance and the capacitance of the silicon dioxide layer. The impedance spectrum of the cellulose acetate coated structure was fitted using the equivalent circuit shown in Fig. 11. The fit results that are summarised in Table 1 confirm the good agreement between the spectra obtained from SPIM and classical AC-impedance spectroscopy. The fit of the impedance spectrum of the coated structure derived from SPIM is shown in Fig. 12. The high frequency part of the spectrum is dominated by the electrolyte resistance and the geometric capacitance and bulk resistance of the polymer. The low frequency part of the spectrum is dominated by the capacitance of the silicon dioxide layer. To increase the sensitivity of the technique and reduce the impact of the substrate on the overall impedance a further reduction in the impedance of the insulator is required.

Table 1

Fit results of impedance spectra for uncoated and coated $\mathrm{SOI} / \mathrm{SiO}_{2}$ structures with a sample area of $7 \mathrm{~mm}^{2}$ obtained from SPIM and classical ACimpedance measurements (FRA)

\begin{tabular}{llllll}
\hline Circuit element & \multicolumn{2}{l}{ Uncoated } & & & \multicolumn{2}{l}{ Coated } & \\
\cline { 2 - 3 } \cline { 6 - 7 } & SPIM & FRA & & SPIM & FRA \\
\hline Electrolyte resistance, $R_{2}(\mathrm{k} \Omega)$ & 6.30 & 2.94 & & 14.4 & 12.1 \\
Oxide capacitance, $C_{1}(\mathrm{nF})$ & 7.29 & 7.04 & & 6.78 & 6.97 \\
Bulk resistance, $R_{1}(\mathrm{k} \Omega)$ & - & - & & 122 & 117 \\
Geometric capacitance, $C_{2}(\mathrm{nF})$ & - & - & & 0.540 & 0.557 \\
\hline
\end{tabular}

Spectra were fitted using the equivalent circuit shown in Fig. 11. 


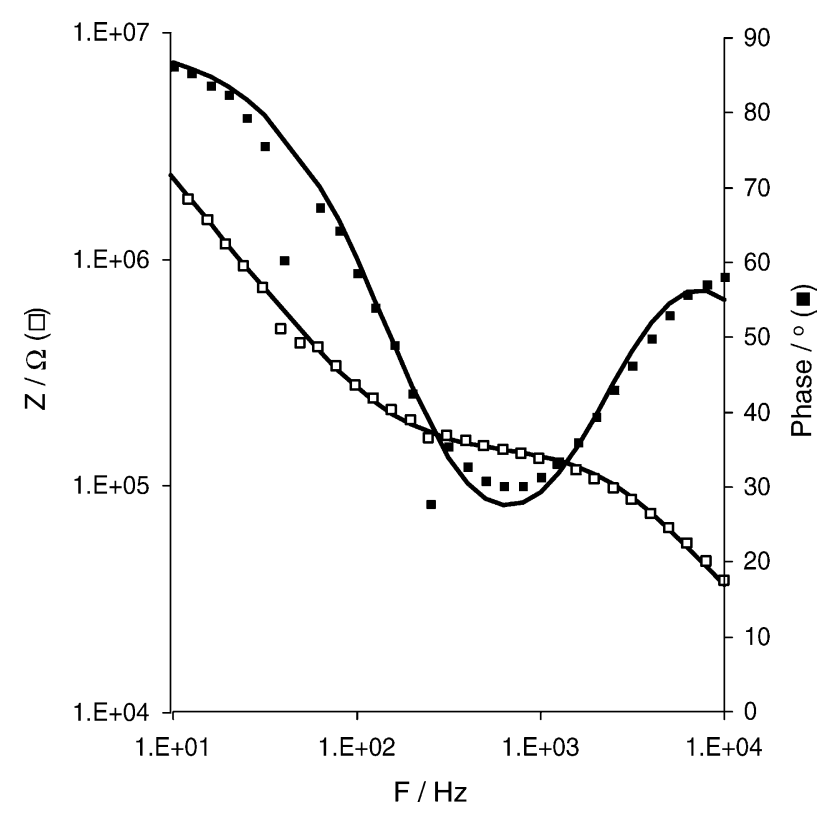

Fig. 12. Fit of the impedance spectrum of a cellulose acetate coated $\mathrm{SOI} / \mathrm{SiO}_{2}$ structure derived from SPIM measurements (dots represent measurement results, lines represent fit using equivalent circuit shown in Fig. 11).

\section{Conclusions}

Using a multiphoton effect for charge carrier generation has resulted in a $31 \%$ improvement of the resolution of photocurrent measurements using bulk silicon as the semiconductor substrate. If the generation of charge carriers had truly been limited to the space charge region, a more significant reduction in the effective diffusion length would have been expected. The relatively small improvement indicates that a significant number of charge carriers was still generated outside the space charge region. Further improvement might be possible using an objective lens with higher numerical aperture as this would cause the local intensity to decrease faster with increasing distance from the focus confining the charge carrier production to an even smaller volume.

Good resolution of photocurrent measurements at fieldeffect structures could be obtained with thin layers of silicon. SOI substrates with a $7 \mu \mathrm{m}$ thick silicon layer were shown to have an effective diffusion length of charge carriers of about $13 \mu \mathrm{m}$. The diffusion length in an SOS substrate with a $1 \mu \mathrm{m}$ thick silicon layer was found to be close to $0.6 \mu \mathrm{m}$. This indicates that SPIM measurements with submicrometer resolution are possible using a high quality optical setup. Considering that George et al. predicted a linear relationship between the thickness of the silicon layer and the resolution of photocurrent measurements, the difference found between SOI and SOS substrates was greater than expected [13]. This may be due to the fact that the silicon layer in case of SOS was not only thinner but also had a higher doping concentration, which is also known to improve the resolution [13].

A drawback of the SOI substrate is that the thin silicon membrane distorts quite easily in contact with an electrolyte solution. This causes problems when an area scan of the photocurrent is to be carried out since the focal distance would not be the same over the entire sample. A current problem associated with field-effect structures based on SOS is, that they have very high flat-band voltages and a larger number of interface states than usually associated with single crystalline silicon. The latter might be caused by mechanical tensions in the material due to different thermal expansion coefficients of silicon and sapphire during the growth of the gate oxide. It is anticipated that the properties of the structure can be improved using a hydrogen treatment.

Using the example of a swelling polymer, cellulose acetate, it was shown that impedance spectra derived from SPIM measurements yield comparable results to spectra measured using a frequency response analyser (FRA). Further improvement of the sensitivity of the technique could be achieved by decreasing the impedance of the gate insulator.

\section{Acknowledgements}

The authors are very grateful to IMRA Lasers for the loan of the Femtolite laser and to Daresbury Laboratory for the use of the Ti-sapphire laser. The authors also wish to thank the Engineering Department at Sheffield University for their support in the fabrication of test structures and the Royal Society and the EPSRC for funding the project.

\section{References}

[1] R.S. Lillard, P.J. Moran, H.S. Isaacs, J. Electrochem. Soc. 139 (1992) 1007.

[2] F. Zou, D. Thierry, H.S. Isaacs, J. Electrochem. Soc. 144 (1997) 1957.

[3] M.M. Lohrengel, A. Moehring, M. Pilaski, Electrochim. Acta 47 (2001) 137.

[4] M. Pilaski, T. Hamelmann, A. Moehring, M.M. Lohrengel, Electrochim. Acta 47 (2002) 2127.

[5] B.B. Katemann, A. Schulte, E.J. Calvo, M. Koudelka-Hep, W. Schuhmann, Electrochem. Commun. 4 (2002) 134.

[6] S. Krause, H. Talabani, M. Xu, W. Moritz, J. Griffiths, Electrochim. Acta 47 (2002) 2143.

[7] O. Engstroem, A. Carlsson, J. Appl. Phys. 54 (1983) 5245.

[8] W.J. Parak, U.G. Hofmann, H.E. Gaub, J.C. Owicki, Sens. Actuators A Phys. 63 (1997) 47.

[9] M. Sartore, M. Adami, C. Nicolini, L. Bousse, S. Mostarshed, D. Hafeman, Sens. Actuators A Phys. 32 (1992) 431.

[10] W. Moritz, I. Gerhardt, D. Roden, M. Xu, S. Krause, Fresenius J. Anal. Chem. 367 (2000) 329

[11] W. Moritz, T. Yoshinobu, F. Finger, S. Krause, M. Martin-Fernandez, M.J. Schöning, Sens. Actuators B Chem. 103 (2004) 436.

[12] M. Nakao, S. Inoue, T. Yoshinobu, H. Iwasaki, Sens. Actuators B Chem. 34 (1996) 234.

[13] M. George, W.J. Parak, I. Gerhardt, W. Moritz, F. Kaesen, H. Geiger, I. Eisele, H.E. Gaub, Sens. Actuators A Phys. 86 (2000) 187.

[14] Y. Ito, Sens. Actuators B Chem. 52 (1998) 107.

[15] C. Xu, W. Denk, Appl. Phys. Lett. 71 (1997) 2578.

[16] C. Xu, W. Denk, J. Appl. Phys. 86 (1999) 2226.

[17] A. Friebe, W. Moritz, J. Appl. Polym. Sci. 51 (1994) 625. 